\title{
Performance Analysis of Stable Election Protocol for Clustered Heterogeneous Wireless Sensor Networks
}

\author{
Neha Rathi ${ }^{1}$ and Partha Pratim Bhattacharya ${ }^{2}$ \\ Department of Electronics and Communication Engineering \\ Faculty of Engineering and Technology \\ Mody Institute of Technology \& Science (Deemed University) \\ Lakshmangarh, Rajasthan - 332311, India. \\ Ineharathi17@gmail.com \\ hereispartha@gmail.com
}

\begin{abstract}
While wireless sensor networks are increasingly equipped to handle more complicated functions, these battery powered sensors which used in network processing, use their constrained energy to enhance the lifetime of the network especially in a heterogeneous settings. Clustered techniques have since been employed to optimize energy consumption in this energy constrained wireless sensor networks. In Classical clustering protocols, equal energy is assigned to all nodes and they cannot take full benefit of the presence of node heterogeneity. SEP, a heterogeneous-aware protocol is used to prolong the time interval before the death of the first node which is crucial for many applications where the feedback from the sensor network must be reliable. The performance of SEP in comparison to LEACH Protocol is analyzed in this paper whose goal is to increase the stable region and as a result decrease the unstable region and improve the quality of the feedback of wireless sensor network, in presence of heterogeneous nodes.
\end{abstract}

\section{General Terms}

Wireless Sensor Network

\section{Indexing terms}

Wireless Sensor Network, advanced nodes, normal nodes, network structure

\section{Council for Innovative Research}

Peer Review Research Publishing System

\section{Journal: INTERNATIONAL JOURNAL OF COMPUTERS \& TECHNOLOGY}

\author{
Vol 6, No 3 \\ editor@cirworld.com \\ www.cirworld.com, member.cirworld.com
}




\section{INTRODUCTION}

A wireless sensor network is composed of numerous sensor nodes which have sensing, computational ability and selforganizing capability. These sensor nodes can sense its periphery to collect the data such as temperature, humidity and even specific events. Each sensor node communicates with other nodes within its radio communication range [1]. These sensors suffer from power limitation because they are deployed in remote places that are not easy to reach. New sensors have to be deployed to replace the old ones because the life time duration of such devices is very limited. It is some of these limitations that has shown an increasing interest from the scientific community to research in such devises that would enhance the longevity and coverage of the devices by using various new technology developments in this field. The main focus is to enhance the life time of sensors and to use the limited resources efficiently by adopting mechanisms, algorithms and protocols that consider these limited resources as main priorities and challenges to produce efficient and reliable networks [2].

The reminder of this paper is divided into five sections. Section II discussed the literature review. Section III shows the heterogeneous WSN model. Performance of LEACH and SEP is reported in section IV and Section V concludes the paper.

\section{LITERATURE REVIEW}

Clustering techniques is used to manage the energy in WSNs. Low Energy Adaptive Clustering Hierarchy $(\mathrm{LEACH})$, a clustering based protocol make use of randomized rotation of cluster-heads to evenly distribute the energy load among the sensors in the network [3]. However, LEACH protocol is not heterogeneity-aware. When the energy is not properly distributed among nodes in the network, the sensors die out faster than they normally should have if they were to maintain their energy uniformly. In real life situation it is difficult for the sensors to maintain their energy uniformly, thus, introducing energy imbalances. LEACH assumes that each node in the network uses equal amount of energy with respect to the overall energy of the system. Conventional protocols such as Minimum Transmission Energy (MTE) and Direct Transmission (DT) [4] do not also assure a balanced and uniformly use of the sensor's respective energies as the network evolves. Stable Election Protocol (SEP) [5], a heterogeneous aware protocol, based on weighted election probabilities of each node to become cluster head according to their respective energy. This approach ensures that the cluster head election is randomly selected and distributed based on the fraction of energy of each node assuring a uniform use of the nodes energy. In the SEP, two types of nodes (two tier in-clustering) and two level hierarchies were considered. SEP prolong the time interval before the death of the first node (refer to as stability period), which is crucial for many applications where the feedback from the sensor network must be reliable.

\section{HETEROGENEOUS WSN MODEL}

In this section, a model of a wireless sensor network in which the nodes are heterogeneous in their initial amount of energy is described. In this model some percentage of the population of sensor nodes assembled with more energy resources than the rest of the nodes. Let $m$ be the fraction of the total number of nodes $n$, which are equipped with $\alpha$ times more energy than the others. These powerful nodes are advanced nodes, and the rest $(1-m) \times n$ are normal nodes. All nodes are distributed uniformly over the sensor field.

\section{Clustering Hierarchy}

A sensor network that is hierarchically clustered is considered here. The LEACH (Low Energy Adaptive Clustering Hierarchy) protocol [6] maintains such clustering hierarchy. In LEACH, random rotation of cluster head is used to evenly distribute the energy among the network. Only the cluster head has to report to the sink and may expend a large amount of energy, but this happens periodically for each node. In LEACH there is an optimal percentage $p_{\text {opt }}$ (determined a priori) of nodes that has to become cluster heads in each round assuming uniform distribution of nodes in space. If the nodes are homogeneous, then in LEACH protocol each node will become a cluster head exactly once every $1 /$ popt rounds. Initially each node can become a cluster head with a probability $p_{\text {opt. }}$ On average, $n \times p_{\text {opt }}$ nodes must become cluster heads per round per epoch. Nodes that are elected to be cluster heads in the current round can no longer become cluster heads in the same epoch. The non-elected nodes belong to the set $G$ and in order to maintain a steady number of cluster heads per round, the probability of nodes $\epsilon G$ to become a cluster head increases after each round in the same epoch. The decision is made at the beginning of each round by each node $s \in G$ independently choosing a random number in [0, 1]. If the random number is less than a threshold $T(s)$ then the node becomes a cluster head in the current round. The threshold is set as:

$$
T(s)= \begin{cases}\frac{p_{\text {opt }}}{1-p_{\text {opt }\left(r \bmod \frac{1}{p_{\text {opt }}}\right)}} & \text { if } s \in G \\ 0 & \text { otherwise }\end{cases}
$$

where $r$ is the current round number. The election probability of nodes $\epsilon G$ to become cluster heads increases in each round in the same epoch and becomes equal to 1 in the last round of the epoch. How the election process of cluster heads should be adapted appropriately to deal with heterogeneous nodes is shown here, which means that not all the nodes in the field have the same initial energy. 


\section{Optimal Clustering}

The clustering is said to be optimal when the energy consumption is well conveyed to all sensors in the network and the total energy consumption is minimum. Such optimal clustering highly rely upon the energy model used here [6].

According to the radio energy dissipation model illustrated in Figure 1, in order to achieve an acceptable Signal-to-Noise Ratio (SNR) in transmitting an L-bit message over a distance $d$, the energy expended by the radio is given by [28]:

$$
E_{T x}(l, d)= \begin{cases}L . E_{\text {elec }}+L \cdot \epsilon_{f s} \cdot d^{2} & \text { if } d<d_{o} \\ L . E_{\text {elec }}+L \cdot \epsilon_{m p} \cdot d^{4} & \text { if } d>d_{o}\end{cases}
$$

where $E_{\text {elec }}$ is the energy dissipated per bit to run the transmitter or the receiver circuit, $\epsilon_{m p}$ and $\epsilon_{f s}$ depend on the transmitter amplifier model we used, and $d$ is the distance between the sender and the receiver. To receive an $L-b i t$ message the radio expends:

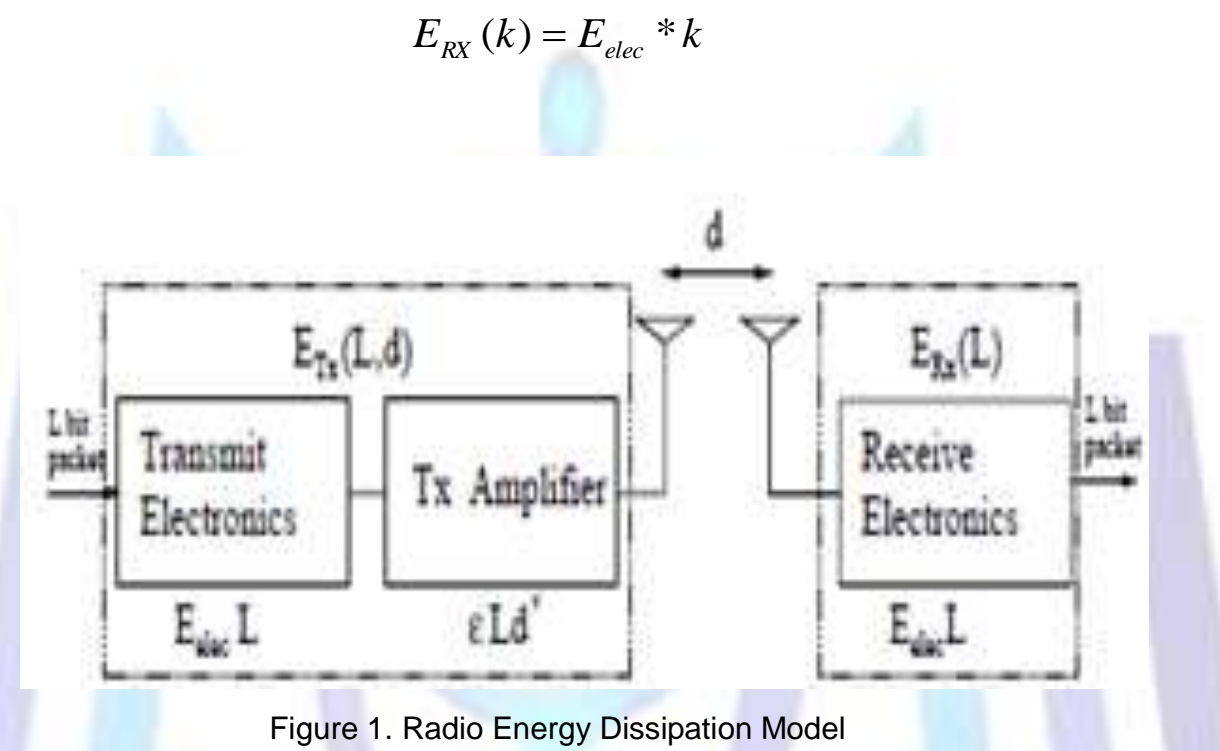

In homogeneous network, unstable region will be very less for LEACH. After the death of the first node, all the remaining nodes are expected to die on average within a small number of rounds as a consequence of the uniformly remaining energy due to the well distributed energy consumption. Even when the system perform in the unstable region, if the spatial density of the sensor network is large, the probability that a large number of nodes be elected as cluster heads is significant for a significant part of the unstable region. In this case, even though the system is unstable in this region, still have a relatively reliable clustering (sensing) process. The same can be noticed when the $p_{\text {opt }}$ is large and spatial density is very low. However, LEACH produce the higher unstable region for heterogeneous network. The reason behind it is that all advanced nodes are equipped with almost the same energy but the election of cluster head is unstable and most of the time these nodes are not used because there is no cluster head to communicate.

This problem of improper cluster head selection is overcome by SEP. SEP enhance the stable region and as a result decrease the unstable region and improve the quality of the feedback of wireless clustered sensor networks, in the presence of heterogeneous nodes.

\section{PERFORMANCE OF SEP AND LEACH}

We simulate a clustered wireless sensor network in a field with dimensions $100 \mathrm{~m} \times 100 \mathrm{~m}$. The population of the sensors is equal to $n=100$ and the nodes, both normal and advanced, are randomly (uniformly) distributed over the field. We placed the BS at a far distance from all other nodes. We simulate the results $100 \mathrm{~m} \times 100 \mathrm{~m}$ plot when our BS is located at (50, 300 ) so that the $B S$ is at least $100 \mathrm{~m}$ from the closest sensor node. The initial energy of a normal node has been set to $E_{0}$ $=0.5 \mathrm{~J}$. The radio characteristics used in our simulations are summarized in Table 1 .

The size of the message that nodes send to their cluster heads as well as the size of the (aggregate) message that a cluster head sends to the sink is set to 4000 bits.

We denote with 'o' a normal node, with '+' an advanced node, with'.' a dead node, with '*' a cluster head and with ' $x$ 'the sink in figure 2. 


\begin{tabular}{|c|c|}
\hline Operation & Energy Dissipated \\
\hline Transmitter/Receiver Electronics & $\mathrm{E}_{\text {elec }}=50 \mathrm{Nj} / \mathrm{bit}$ \\
\hline Data Aggregation & $\mathrm{E}_{\mathrm{DA}}=5 \mathrm{Nj} / \mathrm{bit} / \mathrm{symbol}$ \\
\hline Transmit Amplifier if $\mathrm{d}_{\text {maxtoBs }} \leq \mathrm{d}_{\circ}$ & $\epsilon_{f s}=1 \mathrm{Pj} / \mathrm{bit} / \mathrm{m}^{2}$ \\
\hline Transmit Amplifier if $\mathrm{d}_{\text {maxtoBs }} \geq \mathrm{d}_{\circ}$ & $\epsilon_{m p}=0.0013 \mathrm{Pj} / \mathrm{bit} / \mathrm{m}^{2}$ \\
\hline
\end{tabular}

Table 1. Radio Characteristics used in Simulations

The results of LEACH simulations are shown in Figure 3 for $m=0.1$ and $a=2$ and for $m=0.2$ and $a=3$. It is analyzed that LEACH takes some benefit of the existence of advanced nodes, as the first node dies after a longer stability period when compared to the homogeneous case $(m=a=0)$. It increases the lifetime of the network but throughput is still low. The reason is that after the death of a significant number of nodes, the cluster head election process becomes unstable and as a result less nodes become cluster heads. Even worse, during the last rounds, there are only few rounds where more than one cluster head is elected. Number of alive nodes for the scenario $(m=0.2$ and $a=1)$ and $(m=0.2$ and $a=3)$ is shown in figure 3 (a). From results it is cleared that LEACH fails to take the full advantage of heterogeneity (extra energy) in both scenarios. Figure 3 (b) shows that the number of alive nodes dies quickly in both the scenarios. On the other hand, advanced nodes die inactively or in a very slow fashion shown in figure 3 (c), because they are not taking the full advantage of extra energy of advanced nodes. After the death of normal nodes, advanced nodes are not elected as a cluster head very often because the election of cluster head is not proper and less than the optimal number. The election of cluster head has also become unstable.

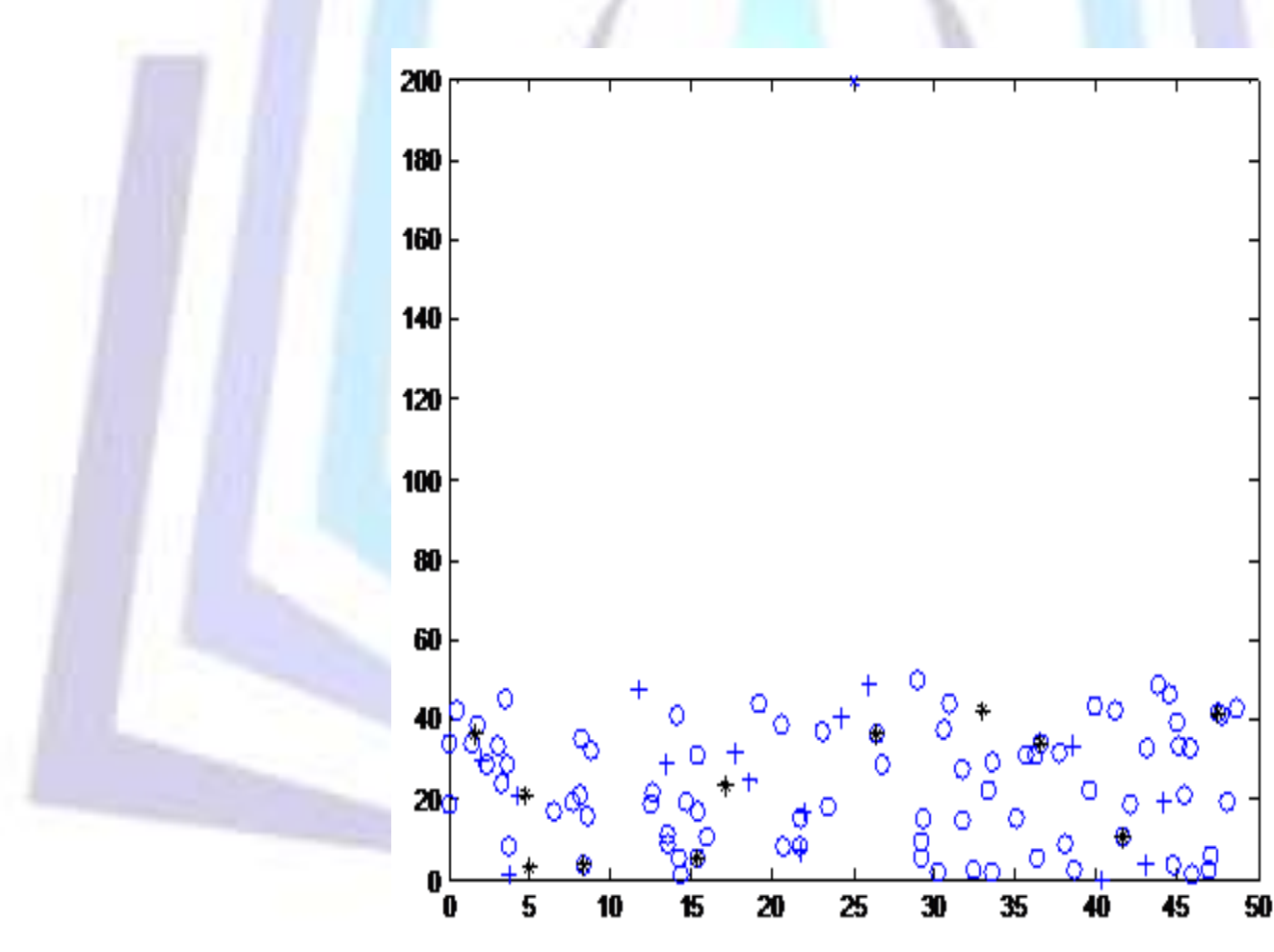

(a) 


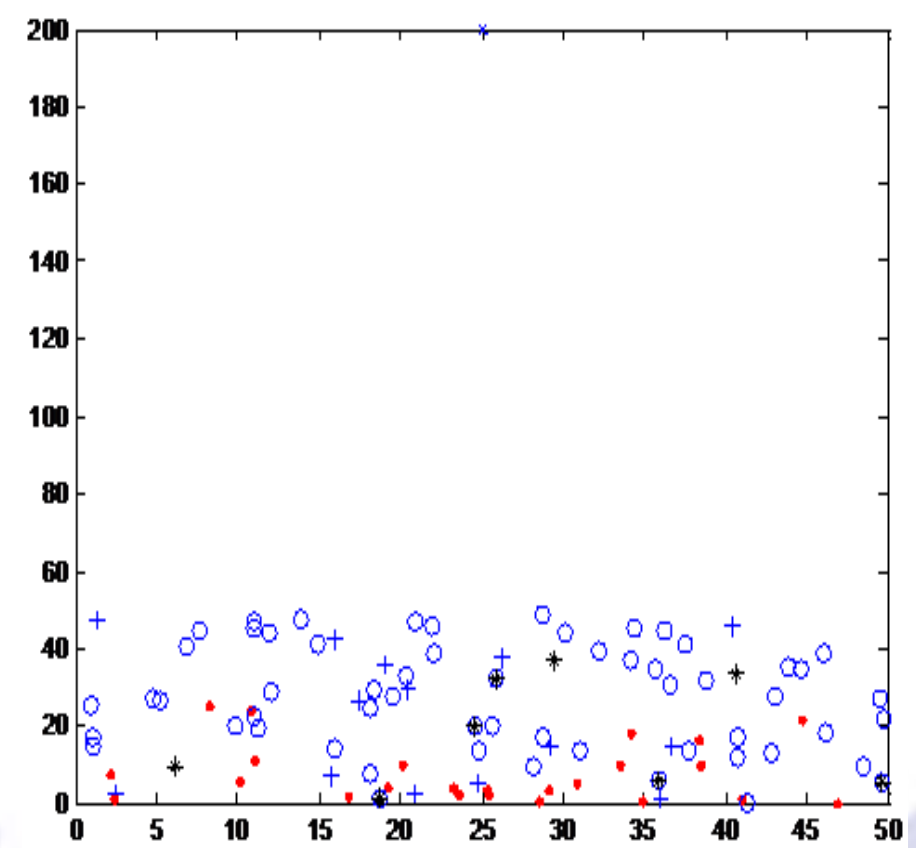

(b)

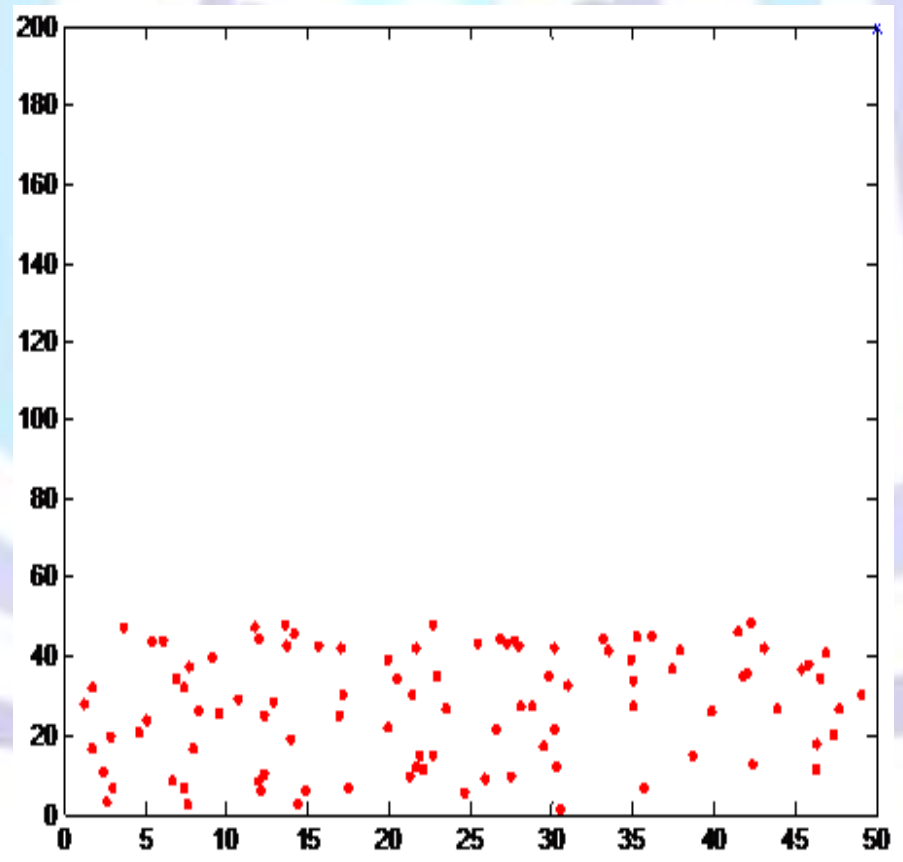

(c)

Figure 2. (a) Heterogeneous Wireless Sensor Network. (b) An instance of the network where some nodes are dead. (c) An instance of the network where all nodes are dead. 


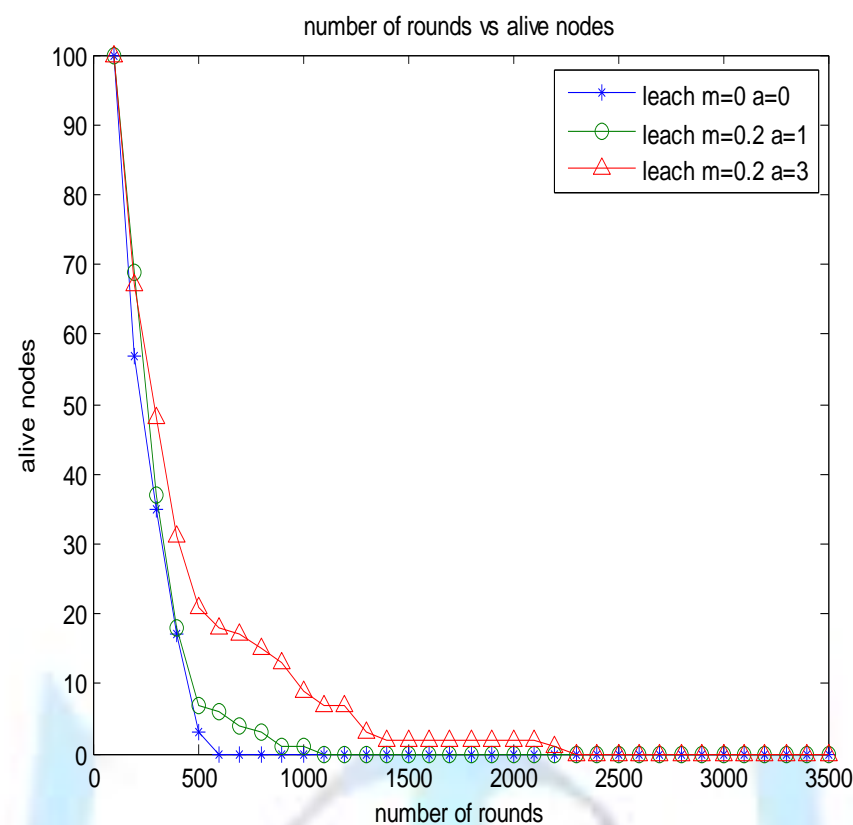

(a)

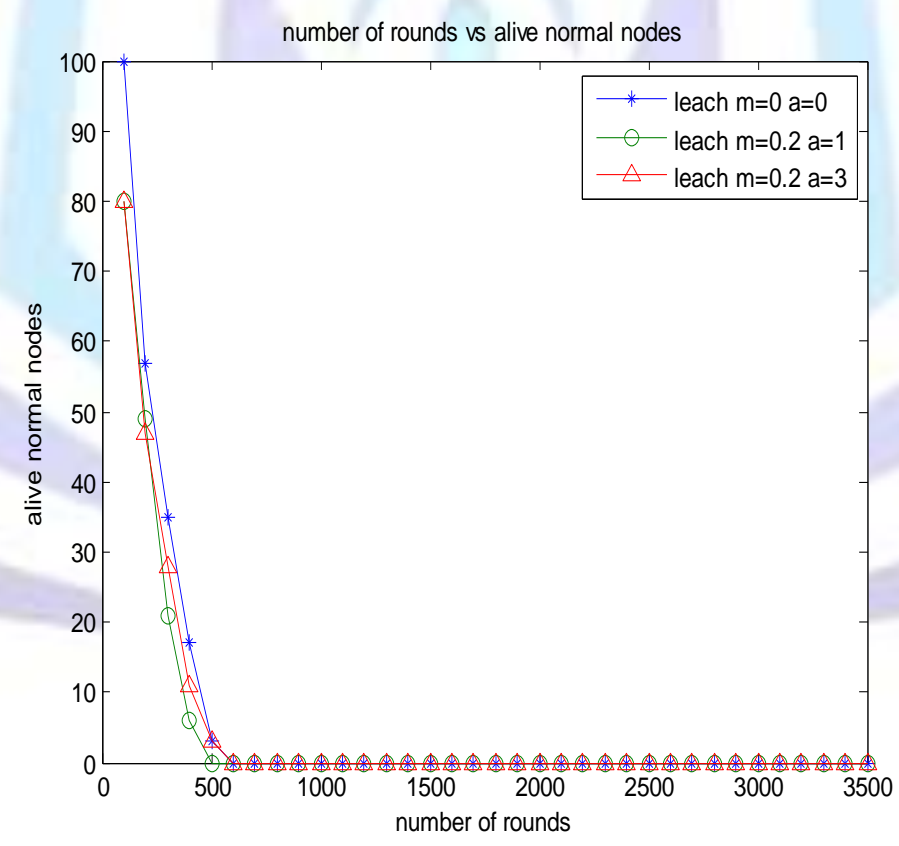

(b) 


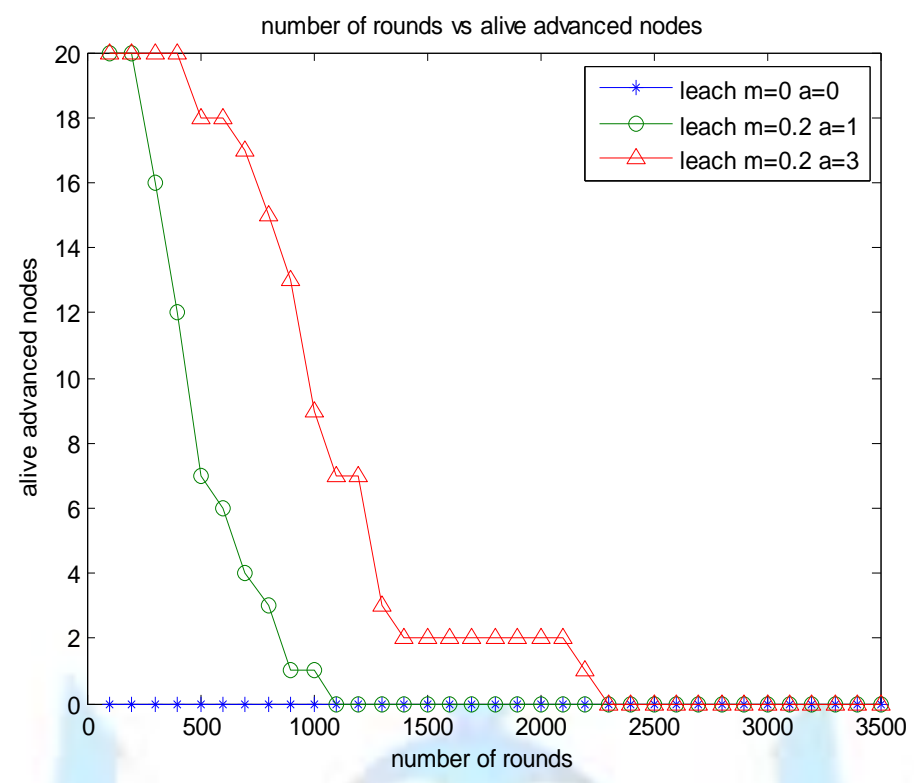

(c)

Figure 3. LEACH behavior in the presence of heterogeneity with $m=0.2$ and $\alpha=3$ : (a) Alive nodes per round; (b) Normal nodes per round; (c) Advanced nodes per round.

Figure 4 shows the performance of SEP Protocol in comparison to LEACH. Figure 4 (a) shows the result for $\mathrm{m}=0.2$ and $\mathrm{a}=1$. It is analyzed from this figure that SEP is taking the full advantage of heterogeneity. The stable region is extended in comparison to LEACH. It is clearly analyzed from figure 4 (b) that the SEP protocol is taking the full advantage of extra energy but advanced nodes of LEACH dies in very slow fashion for the scenario $m=0.2$ and $a=1$. Figure 4 (c) shows that normal nodes dies very fast in both SEP and LEACH. (Figure 4 (d) (e) (f)) shows the same results for the scenario $\mathrm{m}=0.2$ and $\mathrm{a}=3$.

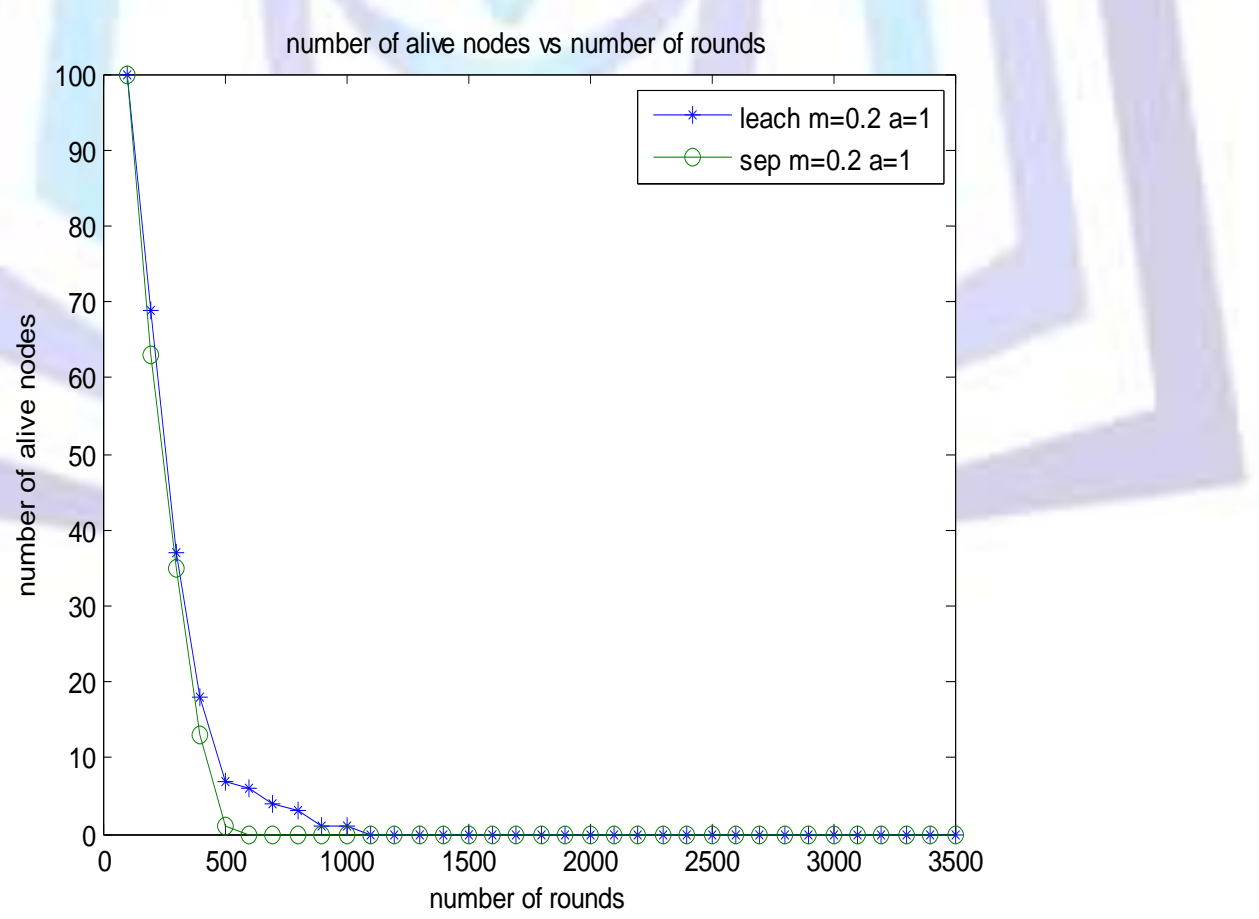

(a) 


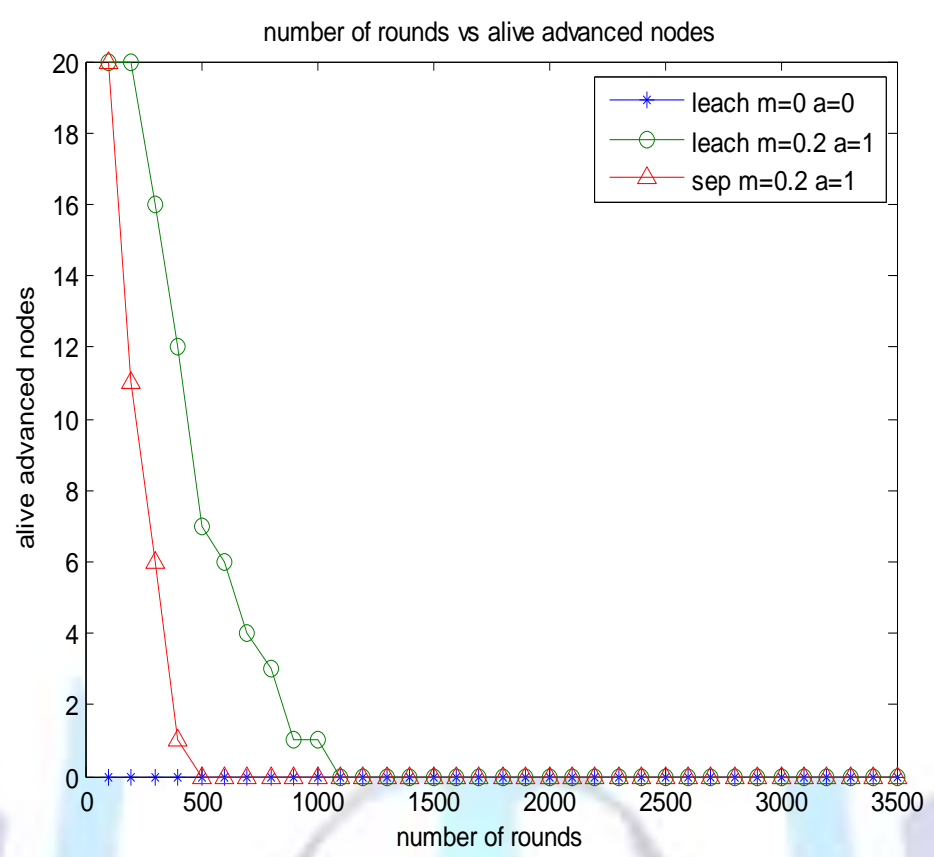

(b)

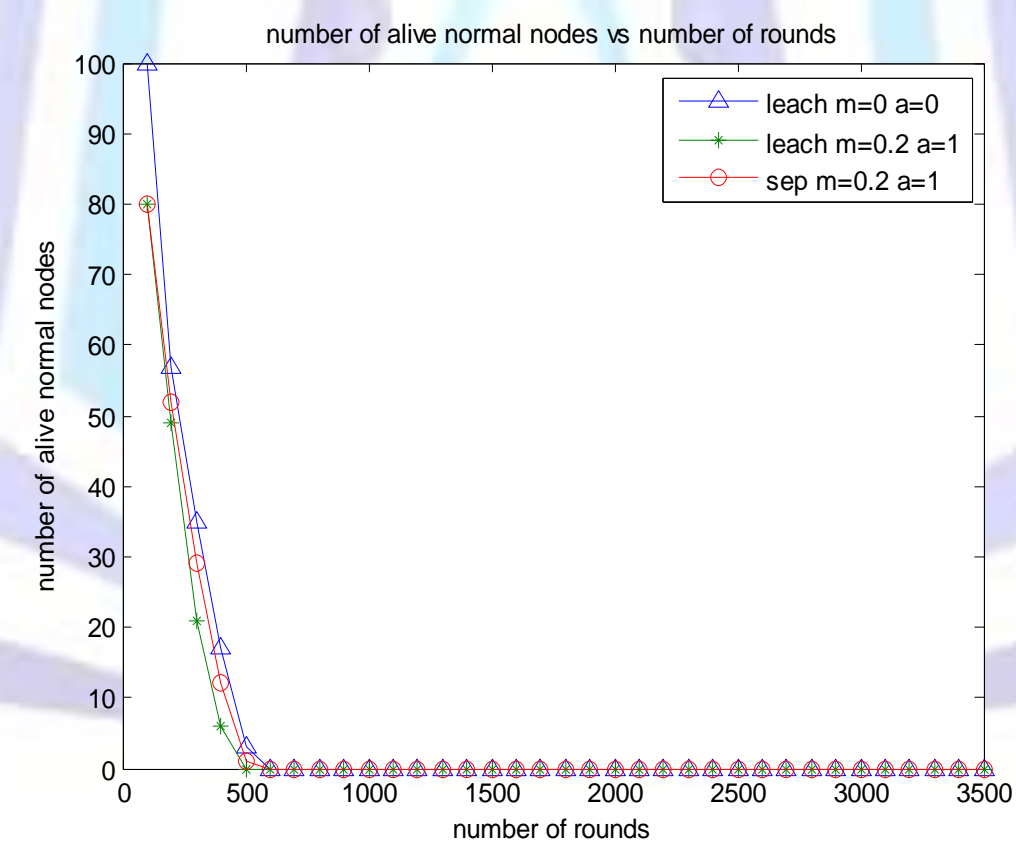

(c) 


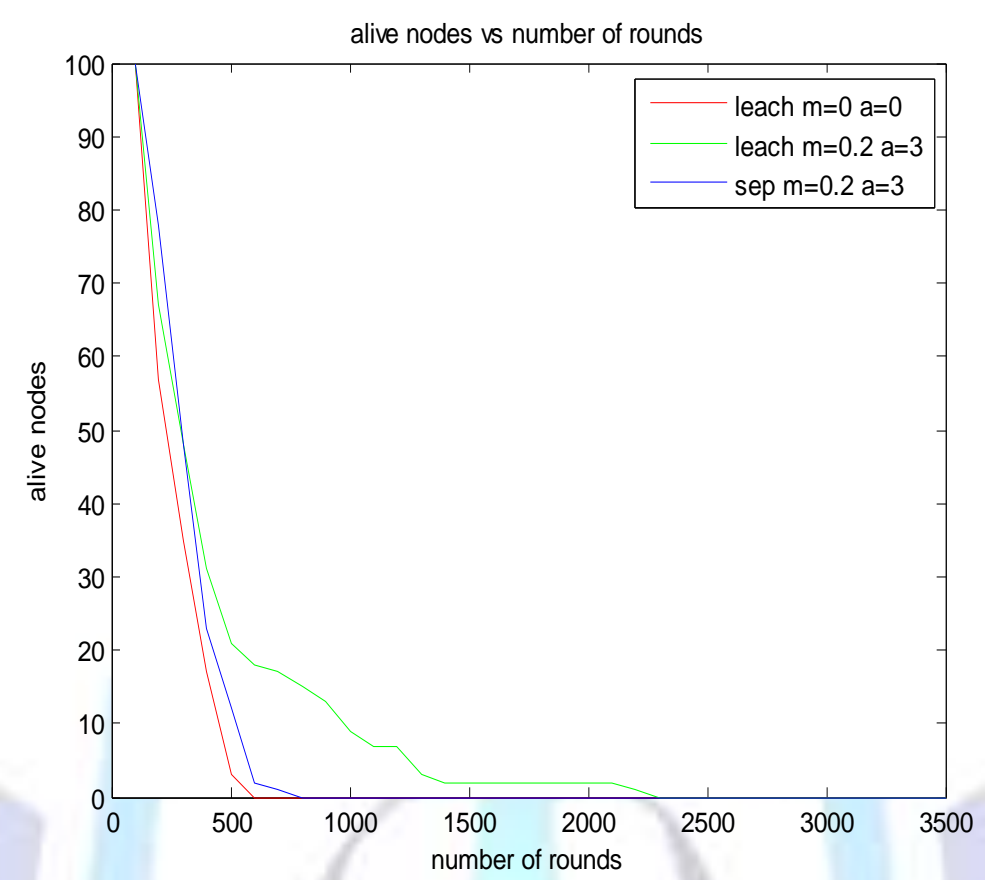

(d)

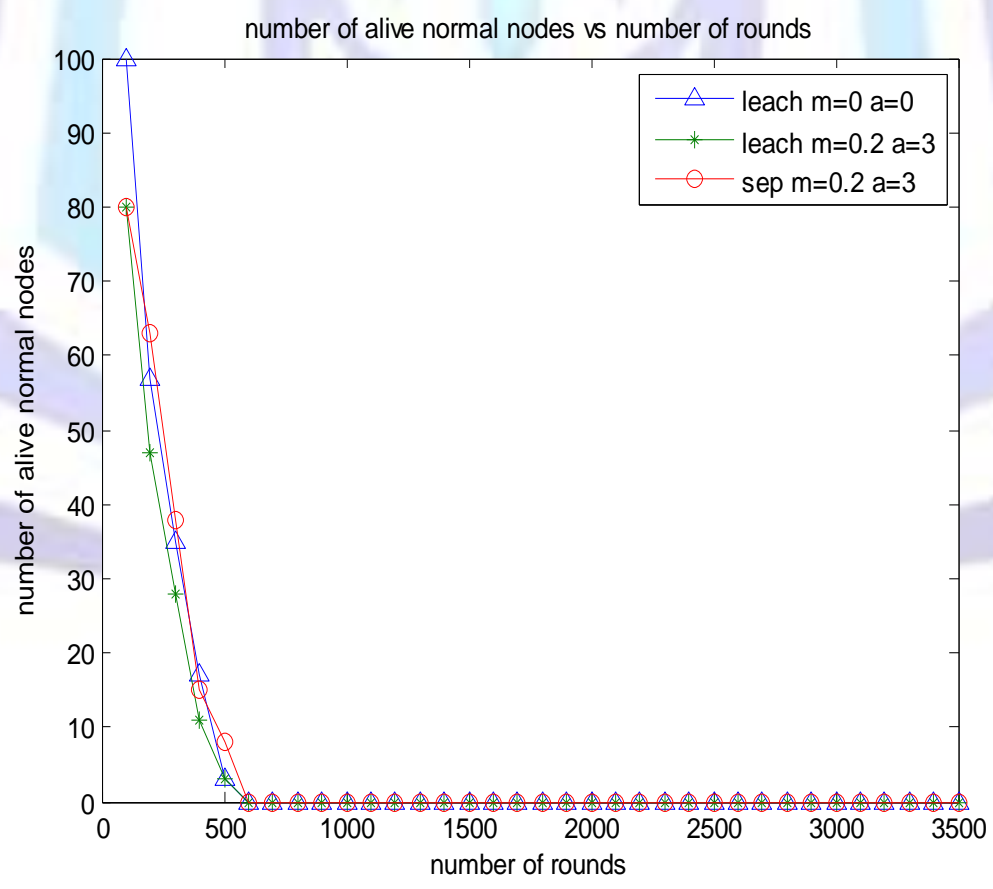

(e) 


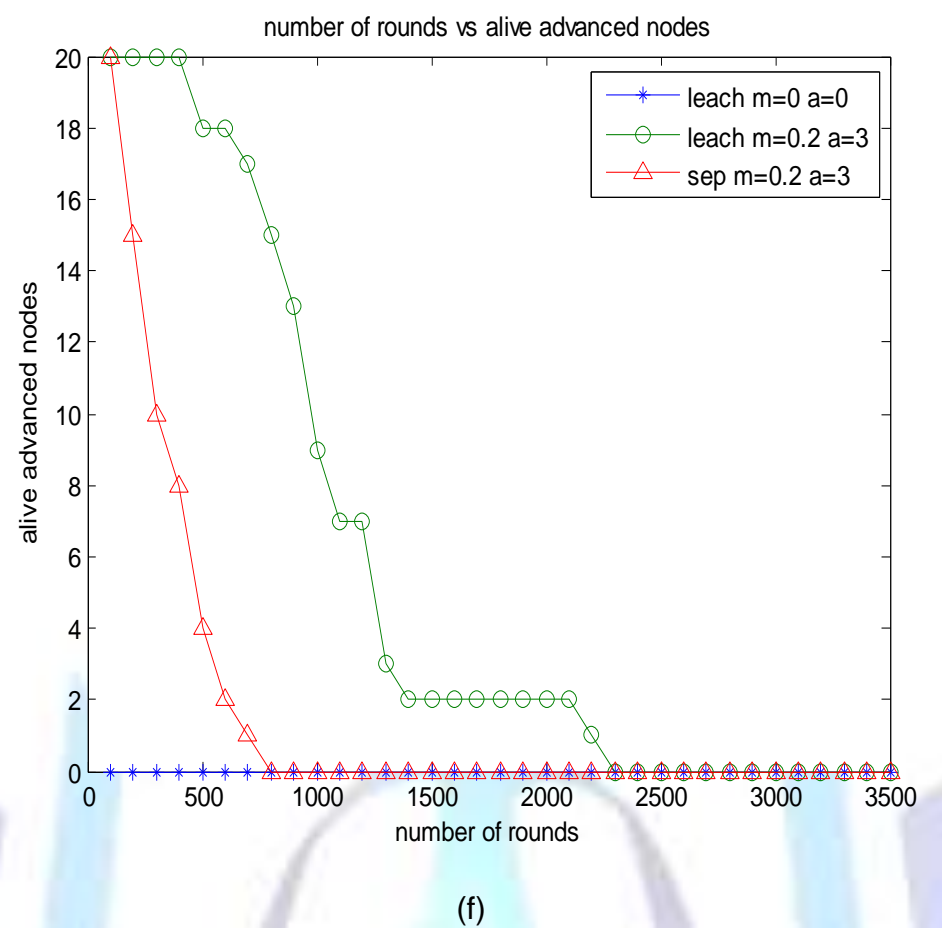

Figure 4. Comparison between LEACH and SEP in the presence of heterogeneity: (a) alive nodes per round for $m=0.2$ and $\alpha=1$; (b) alive advanced nodes per round for $m=0.2$ and $a=1$; (c) alive normal nodes per round for $m=0.2$ and $a=1$ (d) alive nodes per round for $m=0.2$ and $a=3$; (e) alive normal node per round for $m=0.2$ and $a=3$; (f) alive advanced nodes per round for $m=0.2$ and $a=3$

\section{CONCLUSION}

The performance of SEP is analyzed in this paper whose goal is to increase the stable region and as a result decrease the unstable region and improve the quality of the feedback of wireless sensor network, in presence of heterogeneous nodes. The SEP is compared to LEACH protocol. It is analyzed that SEP is taking the full advantage of heterogeneity. The stable region is extended in comparison to LEACH. It is clearly analyzed that the SEP is taking the full advantage of extra energy but advanced nodes of LEACH dies in very slow fashion and normal nodes dies very fast in both SEP and LEACH, which shows that election of cluster heads is proper in SEP when compared to LEACH protocol.

\section{REFERENCES}

[1] Neha Rathi, Jyoti Saraswat and Partha Pratim Bhattacharya, "A Review On Routing Protocols For Application In Wireless Sensor Networks", International Journal of Distributed and Parallel Systems (IJDPS) Vol.3, No.5, September 2012 , pp : 39-58.

[2] S.Prasanna and Srinivasa Rao, "An Overview of Wireless Sensor Networks Applications and Security", International Journal of Soft Computing and Engineering (IJSCE) ISSN: 2231-2307, Volume-2, Issue-2, May 2012.

[3] W. R. Heinzelman, A. P. Chandrakasan, and H. Balakrishnan "An application-specific protocol architecture for wireless microsensor networks," IEEE Transactions on Wireless Communications, vol. 1, no. 4, pp. 660-670, October 2002.

[4] T. J. Shepard, "A channel access scheme for large dense packet radio networks," in Proccedings of ACM SIGCOMM, September 1996, pp. 219-230.

[5] G. Smaragdakis, I. Matta, A. Bestavros, "SEP: A Stable Election Protocol for clustered heterogeneous wireless sensor networks", in: Second International Workshop on Sensor and Actor Network Protocols and Applications (SANPA 2004), 2004.

[6] W. R. Heinzelman, A. Chandrakasan, and H. Balakrishnan, "Energy-efficient communication protocol for wireless micro sensor networks", In Proceedings of the 33rd Hawaii International Conference on System Sciences (HICSS33), January 2000. 


\section{Author' biography with Photo}

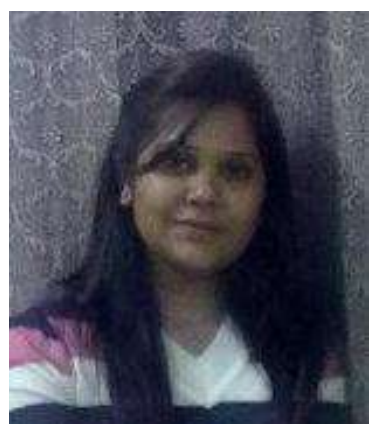

Neha Rathi was born in India on 16 july,1987. She received her B. Tech degree in Electronics and Communication from Uttar Pradesh Technical University, India in 2010 and currently is a M. Tech (Signal Processing) student in Mody Institute of Technology and Science (Deemed University), Rajasthan, India.

Dr. Partha Pratim Bhattacharya was born in India on January 3, 1971. He has 16 years of experience in teaching and research. He served many reputed educational Institutes in India in various positions. At present he is working as Professor in Department of Electronics and Communication Engineering in the Faculty of Engineering and Technology, Mody Institute of Technology and Science (Deemed University), Rajasthan, India. He worked on Microwave devices and systems and mobile cellular communication systems. He has published more than 95 papers in refereed journals and conferences. His present research interest includes mobile cellular communication, wireless sensor network and cognitive radio.

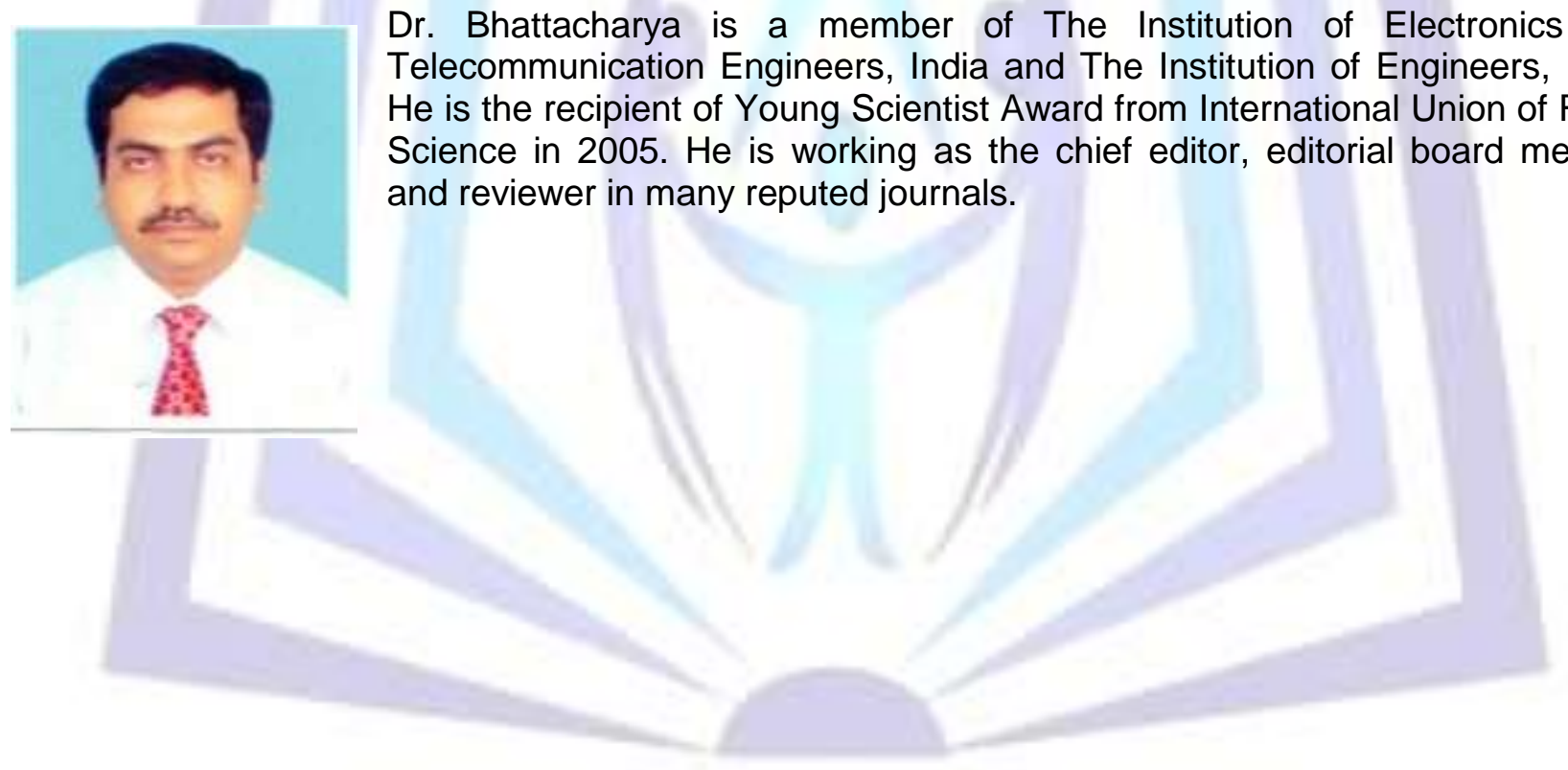

